\title{
LOCAL GRADIENT ESTIMATES FOR THE $p(x)$-LAPLACIAN ELLIPTIC EQUATIONS
}

\author{
FENGPING YAO
}

Abstract. In this paper we give a new and direct proof of local $L^{q}$ estimates for the nonhomogeneous $p(x)$-Laplacian elliptic equation under some proper conditions on $p(x)>1$. We prove that

$$
|\mathbf{f}|^{p(x)} \in L_{l o c}^{q} \Longrightarrow|\nabla u|^{p(x)} \in L_{l o c}^{q} \quad \text { for any } q \geqslant 1
$$

for weak solutions of

$$
\operatorname{div}\left(|\nabla u|^{p(x)-2} \nabla u\right)=\operatorname{div}\left(|\mathbf{f}|^{p(x)-2} \mathbf{f}\right) \quad \text { in } \Omega .
$$

Mathematics subject classification (2010): 35J60, 35J70.

Keywords and phrases: Regularity, $L^{q}$, divergence, quasilinear, elliptic, $p(x)$-Laplacian.

\section{REFERENCES}

[1] E. Acerbi \& G. Mingione, Regularity results for a stationary electro-rheologicaluids, Arch. Ration. Mech. Anal. bf 164, 3 (2002), 213-259.

[2] E. ACERBi \& G. Mingione, Gradient estimates for the $p(x)$-Laplacean system, J. Reine Angew. Math. bf 584 (2005), 117-148.

[3] S. BYUN \& L. WANG, Elliptic equations with BMO coefficients in Reifenberg domains, Comm. Pure Appl. Math. bf 57, 10 (2004), 1283-1310.

[4] S. BYUN \& L. WANG, Quasilinear elliptic equations with BMO coefficients in Lipschitz domains, Trans. Amer. Math. Soc. bf 359, 12 (2007), 5899-5913.

[5] L. A. Caffarelli, X. Cabré, Fully nonlinear elliptic equations, American Mathematical Society, Vol. 43, Providense, RI, 1995.

[6] S. Challal \& A. Lyaghfouri, Gradient estimates for $p(x)$-harmonic functions, Manuscripta Math. bf 131, 3-4 (2010), 403-414.

[7] A. Coscia \& G. Mingione, Hölder continuity of the gradient of $p(x)$-harmonic mappings, C. R. Acad. Sci. Paris Math. bf 328, 4 (1999), 363-368.

[8] E. DiBenedetto \& J. Manfredi, On the higer integrability of the gradient of weak solutions of certain degenerate elliptic systems, Amer. J. Math. bf 115 (1993), 1107-1134.

[9] L. DIENING \& M. RŮŽIČKA, Calderón-Zygmund operators on generalized Lebesgue spaces $L^{p(\cdot)}$ and problems related to fluid dynamics, J. Reine Angew. Math. bf 563 (2003), 197-220.

[10] X. FAN, Global $C^{1, \alpha}$ regularity for variable exponent elliptic equations in divergence form, J. Differential Equations bf 235 (2007), 397-417.

[11] X. Fan, J. Shen \& D. ZhaO, Sobolev embedding theorems for spaces $W^{k, p(x)}(\Omega)$, J. Math. Anal. Appl. bf 262 (2001), 749-760.

[12] X. FAn \& D. ZHAO, On the spaces $L^{p(x)}(\Omega)$ and $W^{m, p(x)}(\Omega)$, J. Math. Anal. Appl. bf 263 (2001), 424-446.

[13] P. Harjulehto, Variable exponent Sobolev spaces with zero boundary values, Math. Bohem. bf 132 (2007), 125-136.

[14] T. IWANIEC, Projections onto gradient fields and $L^{p}$-estimates for degenerated elliptic operators, Studia Math. bf 75 (1983), 293-312. 
[15] J. KinNUNEN \& S. ZHOU, A local estimate for nonlinear equations with discontinuous coefficients, Comm. Partial Differential Equations bf 24 (1999), 2043-2068.

[16] A. Lyaghfouri, Hölder continuity of $p(x)$-superharmonic functions, Nonlinear Anal. bf 73, 8 (2010), 2433-2444.

[17] K. R. RAJAGOPAL \& M. RŮŽIČKA, Mathematical modeling of electro-rheological materials, Contin. Mech. Thermodyn. bf 13, 1 (2001), 59-78.

[18] M. RŮŽIČKA, Electrorheological Fluids: Modeling and Mathematical Theory, Lecture Notes in Math., vol. 1748, Springer, Berlin, 2000.

[19] E. Stein, Harmonic analysis, Princeton University Press, Princeton, NJ, 1993.

[20] L. WANG, A geometric approach to the Calderón-Zygmund estimates, Acta Math. Sin. (Engl. Ser.) bf 19, 2 (2003), 381-396. 